hypothyroidism was higher than that of subclinical hyperthyroidism in this study population. TSH levels were positively and linearly associated with the TC and TG levels in euthyroid non-diabetics with newly diagnosed asymptomatic CHD. Within the normal range, TSH might exert direct and positive effect on TC and TG, and the influence on TC was stronger than that on TG.

\section{GW23-e1403 RELATIONSHIP OF SERUM TSH WITH LIPID PROFILE IN PATIENTS WITH NEWLY DIAGNOSED ASYMPTOMATIC CORONARY HEART DISEASE}

doi:10.1136/heartjnl-2012-302920j.5
Xing Wanjia, Zhao Jiajun. Provincial Hospital Affiliated to Shandong University

Objectives Thyroid status may affect lipid profile and the mechanism of this relationship is traditionally attributed to the influence of thyroid hormone. Some recent study indicated that TSH might up-regulate the key enzyme in cholesterol synthesis and have a role in maintaining lipid homeostasis. The goal of this study was to probe into the relationship between serum TSH levels and the lipid profile in patients with newly diagnosed asymptomatic coronary heart disease (CHD).

Methods A retrospective study was conducted including 689 patients with newly diagnosed asymptomatic CHD from 2004 to 2010 in Jinan, China (308 males and 381 females, age ranged 45-88 years). Lipid parameters and the levels of TSH, thyroid hormones were determined. Patients were grouped into different thyroid status according to the thyroid function assay. Multivariate regression analysis was used to assess the association between TSH level and lipid profile. Path model analysis was employed to determine the total effect, direct and indirect effect of TSH on lipid parameters.

Results Most of the patients were euthyroid (78.4\%), while the prevalence of thyroid dysfunction including subclinical ones was $21.6 \%$. $\mathrm{SCH}$ accounted for $8.6 \%$, while subclinical hyperthyroidism accounted for only $1.5 \%$. There were more patients with hypothyroidism than those with hyperthyroidism including the subclinical ones (14\% vs $7.7 \%$ ). In euthyroid patients, the TSH level within the normal range was positively and linearly correlated with log-transformed total cholesterol (TC) and triglycerides (TG) $(\beta=0.191$ and 0.113 , respectively; $p<0.001$ and $p<0.05$, respectively). The analysis based on the equations in path model analysis showed that the total effects of TSH on Log TC and Log TG were significant (path coefficient $=0.2028$ and $0.1138, t=4.5037$ and 2.5028 , respectively) in euthyroid patients with newly diagnosed asymptomatic CHD. The results of effects analysis showed that the total effects of TSH on TC and TG were 0.1936 and 0.1095 , respectively. The direct effect of TSH on Log TC and log TG were 0.2028 and 0.1138 , respectively. The indirect of TSH on Log TC and log TG were negative, which were caused by the direct influence of thyroid hormone.

Conclusions Most of the patients with newly diagnosed asymptomatic $\mathrm{CHD}$ were euthyroid, while the prevalence of subclinical 